que celte seconde grille n'est pas indispensable que je ne l'ai pas fait figurer en face de chaque panneau de grille fine.

Le projet, tel qu'il est sur le croquis, prévoit l'emploi, en outre de tous les panneaux des grilles de pẻche, d'un panneau supplémentaire rectangulaire de chaque type : il n'est pas mauvais, en effet, d'en être muni en cas d'avarie. Bien entendu, le nombre des panneaux à installer dépend de l'imporlance du débouché qui est nécessaire à l'évacuation des caux, d'après l'importance du bassin d'alimentation de l'étang.

Le lecteur aura peut-être un certain étonnement à voir étudier en même temps la question de la pêche et celle du déversoir; il a pu se rendre compte de la relation qui existe, sur certains points, entre les deux questions, du fait de l'utilisation des grilles, dont la fabrication est coûteuse, surtout quand ce n'est pas d'un type de séric, utilisation, dis-je, à deux emplois différents, et jamais simultanés, car on ne pèche pas lorsque l'étang est rempli, et le déversoir est à sec lorsque le moment de la pêche approche.

\title{
L'EXPLOITATION DES ÉTANGS EN ALLEMAGNE
}

\author{
Par M. H.-E. DE HEEMSKERCK \\ Vice-Président et Secrétaire génèral \\ du Verband Deutscher Karpfen und Schleienproduzenten, à Breslau.
}

La crise de mévente qui, s'élant accentuée spécialement au cours de l année dernière, éprouve toutes les entreptises piscicoles, nous contraint à rechercher sérieusement les causes de son développenent aussi inquiétant que funeste. Et ce n'est pas à l'Illemagne seule que s'imposent semblables investigations, clles intéressent exactement de mìme tous les autres pays de l'Europe qu'affectent les conditions dans lesquelles s'opère, sur le marché allemand, le débit de la larpe et de la Tanche. Il est évident que nul pays ne saurait, de nos jours, se payer le luxe de méconnaître l'existence de rapports très étroits dans la vie économique internationale et d'en contempler le développement en se plaçant à un point de vue unilatéral êt égoïste.

Ciest donc bien chaleureusement que nous félicitons la France, - convaincue comme elle l'est de la nécessité d'entente internationale en matière économique, - de son intention de faire examiner, par le Congrès international d'aquiculture qui se tiendra à Paris en Juillet, les périls dont est menacée la carpiculture et d'inviter, en première ligne, les pisciculteurs praticiens a y exposer leurs idées. Le fait que la présidence de la sous-section "Cypriniculture " se trouve confiée d̀ un confrère aussi expert et énergique que M. le comte DE Neurbourg, nous donne toute 
garantie, que cetle sous-section là ia réellement faire de la bonne et lucrative besogne.

Mais, anant de nous oceuper du délail des problèmes à résoudre par cetle assemblée, il nous parait utile d'informer sommairement les lecteurs du

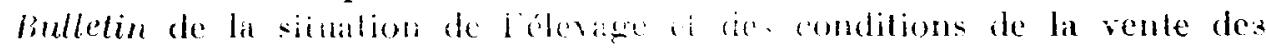

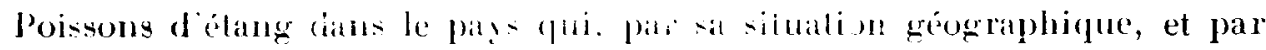
le fait que jusqua présent il ab-orbail ausi un: larere partie de la production de ses voisins. mérite une attention toute spéciale : il sagit de l. lliemagne.

La Carpiculture y représente une branche, très importante dans certaines régions, de lagrriculture. La surface d'eau globale qui y est consacrée s'élève à environ 60.000 hectares, dont fo.000 en Prusse, ro.000 en Bavière, s.ovo en Saxe et 3.000 pour le reste du pays.

La production annuclle varie : - pour la Carpe de consommation, de

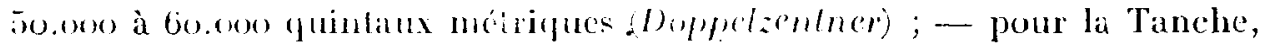
de 5.000 à 6.500 quintaux métriques.

Le territoire de production le plus important est la silésic qui comprend à peu près le liers de la lotalité de la surfice en eau et fournit, par conséquent, le tiers de la peoduction kolale. On se sert presquexchusivement d'étangrs artificiels à déversoir d'can, antínagés en partie dès les $\mathbf{x}^{\circ}$ ou $x \mathbf{r}^{\mathrm{e}}$ siècles. La plupart des exploitant- de quelque importance élèvent leur alevin, tandis que les pisciculteurs moins considérables se contentent d'acheter de la Cirpe de étés au prinlemps pour en faire du P'oisson comestible of rendable ì l'automne.

la gène économique générale ol less conditions défarorables à la production, causées surlout par des difficultés d eau et de climat, ont forcé le pisciculteur allemand, il a a bon nombre d'années léjà, à comprimer autant que possible ses frais de production toul en intensitiant le rendement (engrais, nourrissage, faucardement, ete...). Les risques à courir s'en trouvaient accrus, mais il fallait s'y soumettre.

Conformément à la remante, on éleve la limpe niroir dite "Gaticienne ", qui a remplacé presque completement la Carpe écaille " hausitz "d'autrefois. Jans certaines régions, la larpe cuir " lauzitz" a retromsé des amaleurs daus ress dernicrs lemps.

L'élerage de la Tinche ne forme, en Silérie, quine branche annexe de la Carpiculture; jusqu à préseut on a fait rle la Tanche a consommation de 250 à 500 grammes, mais la demande de sujels de joo a jou grammes commence à prévaloir.

Toutes les observations concordent sur ce point que le consommateur allemand marchambant Carpe on Tamche est devenu bien plus rigoureux

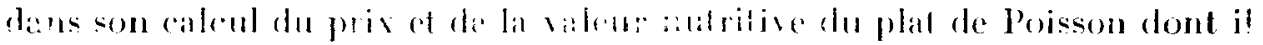
désire faire emplette; $c$ est un signe très remarquable du déclin du pouroir d'achat de la population. Il y a la un fait quion ferail bien de ne pas negliger à l'étrangere avant de cherclier is cyporter ins' l' lllemagne.

Pour éconler la production allemande, on procède comme suit :

Environ $70 \%$ du Poisson d étangr pariennent au consommateur par 
lintermédiaire du commerce de wros el de détail ; 30\% directement par vente locale. On wit, par là, que le role des poissonniers allemands est d’importance considérable. Pour tansporter la marchandise de la station du pisciculteur jusqu’au petit détaillant les marchands de gros se servent de wagrons spéciaux contenant de jo à 150 quintaux métriques. La plupart d'entre eux sont à mème d'entreposer de 35o à 5.000 Q. II. durant un certain temps. Il va sans dire que, de mème, tout producteur un tant soit peu important dispose des viviers nécessilires pour conserver son Poisson pendant l'hiver jusqu à la vente.

Parmi les petits puissonniers, il ny cu a guère plus de 20 à $25 \%$ qui disposent des installations requises pour le débil du Poisson vivant. La raison eil est, que, pour vender de la marée, du Poisson salé ou fumé, etc., nul aménagement spécial ne s impose ; leur commerce est donc beaucoup plus facile, tandis que celui du Poisson vivant exige des bassins spéciaux dont l'alimentation d'eau coûte fort duer en ville. C'est pourquoi, pendant les dernières années, les organisations piscicoles se sont données beaucoup de mal pour éclairer les petits marchands ; une propagande assidue a été faite pour leur démontrer la nécessité pressante de se pourvoir des installations indispensables au débit du Poisson vivant. Pour faciliter cette réforme, on a mème songé à faire meitre les bassins nécessaires à la disposition de certains marchands notables par les grands exploitants eux-mêmes. Ces derniers prèteraient ainsi le matćriel à leurs acquéreurs ; l'idée n'a pas encore été réalisée, mais elle paraît digne de considération.

Les bouleversements engendrés par la gruerre dans les relations économiques entre pays d'Europe n ont pas manqué d'influer aussi sur la production piscicole allemande, en mettant surtout un terme à la stabilité des prix, précédemment régnante. Depuis des années, dans le courant de la première semaine de Septembre, producteurs et marchands allemaands se rassemblent à Cottbus pour délibérer sur la fixation d'un prix directeur prour la vente de la campagne à venir. Jusqu'en rgi3, la plus grande partic de la production d'Allemagne se cendail à la Foire de Cottbus à un prix déterminé à l'avance, mais, depuis, le manque de sécurité dans les transactions a incité les marchands à la plus grande réserve. Il est impossible d'exposer en quelques lignes toutes les causes, qui, d'année en année, ont fait dépérir le commerce de la Carpe ; bornons-nous à constater, que pisciculteurs et marchands souffrent également de la volatilisation presque totale de Jeurs rapilaur d'exploilalion ; que les uns el les autres sont paralysés par le besoin de crélit. Tinsi, de charfue crite est-ent contraint de prendre des mesures dont pàtit forcément la marche normale des affaires. N'oublions pas non plus que l'importation de Carpes comestibles achetées à l'étranger, depuis $\mathrm{s} 9 \%$, a augmenté de plus de $\mathbf{1} 5.000$ Q. M. et cela à des prix qui font le plus grand tort au producteur allemand. Cette dernière circonstance nous paraît avoir été la cause essentielle du récent effondrement des prix, à raison de l'inséculté qui en est résultée pour le marché allemand. 
La progression effrayante de la criso ag̣ricole en lllemag̣ne avait déjà fait

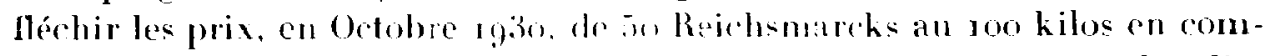
paraison des prix de lannée précédente. Lélranger offrant sa marchandise à un prix encore plus bas, nous avons malheureusement vu s'accentuer encore la baisse. Fincore faut-il observer que. pour cette année-ci, gràce à la collaboration lovale de lous cew qu jntéresse le commerce de la Carpe en tllemagne. le danger ́́norme du dumping russe a pu eitre conjuré. L agence commerciale soviétigue it Berlin avait offert aux marchands allemands environ t.0oo quintaux mátriques de Carpe visante comestible, de provenance russe, au prix de sí R. II. les 100 kilos lis rés frontière. C"était 6o R. II. de moins que ne se inndait à rette époque la Carpe en Allemagne et 20 R. M. en-dessous des prix de France :

Sous l'influence de rese causes de dépression. si elles devaient durer, le marché allemand irait tout droit à sa ruine ; il est difficile de croire que cette ruine pourrait laisser indifférent l'étranger. Yous applaudissons done chaudement le Président de la sous-section de Cypriniculture du Congrè̀s de Paris d'attirer l'attention des Congressistos sur la question suivante :

"Existe-t-il, en Furope, excès de produrlion de Carpe, ou bien est-ce seulement l’irrégularité des débouchés qui produit des débordements avant pour suite crise de mévente et baisse de prix?"

L'attitude que les nations différentes prendront vis-ì-vis de cette question sera décisive pour la marche des travaux du Conerès. Si l'on arrivait à constater un excès de produclion en Furope. il serait bien difficile d'y remédier promptement. car, pour détermincr la nature of l'importance des mesures à prendre : - restriction do la production totale et répartition du restant sur les pays différents - il faudrait d'abord des investigations très minutieuses. Mais il y a une décision que le Congrès ne devrait pas tarder à prendre, en cas d'excès de production reconnue, c'est la résolution d'opposer à ce danger immédiat le recours à des moyens ínergiques pour développer la comsommation du Poisson d'étang.

Si. par contre. la supposition d'un excès de production ne se trowait pas fondé ef que lion crut possible de romédier à la crise de mévente au moyen d'un accord international sur l'écoulement ef les débouchés de la production européenne, le Congrès sera parfaitement en état de trower ce remède. Il suffit que chacpue pays ait soigneusement rassemble la documentation permettant de répondre au questionnaire qui accompaønait l'invitation. Le programme du Congrrès nous laissera tout le temps qu'il faut soit pour lélibérer, soit pour agrir. Il importi que tous les congressistes soient fermement comvancus. il sagit de problemes que seule l'óconomie privée est en état de résoudre ef toute question de politique rommerciale doit etre exclue des délibérations. comme concernant uniquement les gouvernements. Mais si le problème est vigoureusement abordé du point de vue réaliste et technique, il est bien permis d'espérer que les délibérations de la sous-section de "Cypriniculture "auront un résultat supérieur à colui qu'ont eu tant d'autres Congrès internalionaux. 